\title{
APPROXIMATE POINT SPECTRUM AND COMMUTING COMPACT PERTURBATIONS
}

\author{
by VLADIMIR RAKOČEVIĆ
}

(Received 26 June, 1985)

1. Introduction and preliminaries. Let $X$ be an infinite-dimensional complex Banach space and denote the set of bounded (compact) linear operators on $X$ by $B(X)$ $(K(X))$. Let $\sigma(A)$ and $\sigma_{a}(A)$ denote, respectively, the spectrum and approximate point spectrum of an element $A$ of $B(X)$. Set

$$
\begin{aligned}
& \sigma_{e m}(A)=\bigcap_{K \in K(X)} \sigma(A+K), \\
& \sigma_{e a}(A)=\bigcap_{K \in K(X)} \sigma_{a}(A+K), \\
& \sigma_{e b}(A)=\bigcap_{\substack{A K=K A \\
K \in K(X)}} \sigma(A+K), \\
& \sigma_{a b}(A)=\bigcap_{\substack{A K=K A \\
K \in K(X)}} \sigma_{a}(A+K),
\end{aligned}
$$

$\sigma_{e m}(A)$ and $\sigma_{e b}(A)$ are respectively Schechter's and Browder's essential spectrum of $A$ ([16], [9]). $\sigma_{e a}(A)$ is a non-empty compact subset of the set of complex numbers $\mathbb{C}$ and it is called the essential approximate point spectrum of $A([\mathbf{1 3}],[\mathbf{1 4}])$. In this note we characterize $\sigma_{a b}(A)$ and show that if $f$ is a function analytic in a neighborhood of $\sigma(A)$, then $\sigma_{a b}(f(A))=f\left(\sigma_{a b}(A)\right)$. The relation between $\sigma_{a}(A)$ and $\sigma_{a b}(A)$, that is exhibited in this paper, resembles the relation between the $\sigma(A)$ and the $\sigma_{e b}(A)$, and it is reasonable to call $\sigma_{a b}(A)$ Browder's essential approximate point spectrum of $A$.

Throughout this paper $N(A)$ and $R(A)$ will denote respectively the null space and the range space of $A$. Set $\alpha(A)=\operatorname{dim} N(A)$ and $\beta(A)=\operatorname{dim} X / R(A)$. An operator $A \in B(X)$ is called semi-Fredholm if $R(A)$ is closed and at least one of $\alpha(A)$ and $\beta(A)$ is finite. For such an operator $A$ we define an index $i(A)$ by $i(A)=\alpha(A)-\beta(A)$. Let $\Phi_{+}(X)$ denote the set of semi-Fredholm operators with $\alpha(A)<\infty$, and $\Phi_{+}^{-}(X)=\left\{A \in \Phi_{+}(X): i(A) \leqslant 0\right\}$. Then $\sigma_{e a}(A)=\left\{\lambda \in \mathbb{C}: A-\lambda \notin \Phi_{+}^{-}(X)\right\}\left(\left[14\right.\right.$, Theorem 3.1]). Let $\left(G_{n}\right)$ be a sequence of compact subsets of $\mathbb{C}$. The limit superior, lim sup $G_{n}$, is the set of all $\lambda$ in $\mathbb{C}$ such that every neighbourhood of $\lambda$ intersects infinitely many $G_{n}$. A mapping $\tau$ defined on $B(X)$ whose values are compact subsets of $\mathbb{C}$ is said to be upper semi-continuous at $A$ when if $A_{n} \rightarrow A$ then $\lim \sup \tau\left(A_{n}\right) \subset \tau(A)([11])$. The polynomial hull $\hat{E}$ of a compact subset $E$ of the complex plane $\mathbb{C}$ is the complement of the unbounded component of $\mathbb{C} \backslash E$. Given a compact subset $E$ of the plane, a hole of $E$ is a component of $\hat{E} \backslash E$. If $F$ is another compact set such that $\partial E \subset F \subset E$, it follows that $\partial E \subset \partial F, \hat{E}=\hat{F}$ and $E$ can be obtained from $F$ by filling in some holes of $F$. (Here and in what follows $\partial E$ denotes the boundary of the set $E[\mathbf{1 5}]$.) Finally $a(A)$, the ascent of $A$, is the smallest non-negative integer $n$ such that $N\left(A^{n}\right)=N\left(A^{n+1}\right)$. If no such $n$ exists, then $a(A)=\infty$.

Glasgow Math. J. 28 (1986) 193-198. 


\section{Characterization of $\sigma_{a b}(A)$.}

Theorem 2.1. $\lambda \notin \sigma_{a b}(A)$ if and only if $A-\lambda \in \Phi_{+}^{-}(X)$ and $a(A-\lambda)<\infty$.

Proof. If $\lambda \notin \sigma_{a b}(A)$, there is a $K \in K(X)$ such that $A K=K A$ and $\lambda \notin \sigma_{a}(A+K)$. In particular, $A+K-\lambda \in \Phi_{+}^{-}(X)$ and $a(A+K-\lambda)=0$. Adding the operator $-K$ to $A+K-\lambda$, we see that $A-\lambda \in \Phi_{+}^{-}(X)$ ([7, Theorem 5.26 of Chapter IV]) and $a(A-\lambda)<\infty$ ([3, Theorem 2]). To prove the converse suppose that $A-\lambda_{0} \in \Phi_{+}^{-}(X)$ and that $a\left(A-\lambda_{0}\right)<\infty$. If $\lambda_{0} \notin \sigma(A)$, then $\lambda_{0} \notin \sigma_{a b}(A)$ and the proof is complete. Suppose that $\lambda_{0} \in \sigma(A)$. Then $\lambda_{0}$ is an isolated point of $\sigma_{a}(A)\left(\left[10\right.\right.$, Lemma 2.5]). Now $0<\alpha\left(A-\lambda_{0}\right)<$ $\infty$ implies that Kato's number $v\left(A-\lambda_{0}: I\right)$ is finite ([6, Theorem 3]). Following Zemánek's method of removing jumping points ([18, Theorem 7.1]), while applying Kato's reduction theorem ([6, Theorem 4]), we conclude that the space $X$ decomposes into a direct sum of two closed subspaces $X_{0}$ and $X_{1}$. These subspaces are $\left(A-\lambda_{0}\right)$-invariant, hence $A$-invariant, and have the following properties: (we quote only those relevant to our problem). The space $X_{1}$ is finite dimensional (and $A-\lambda_{0}$ is nilpotent on it). If $A_{0}$ is the restriction of $A$ to $X_{0}$ considered as an operator from $X_{0}$ into itself then $\alpha\left(A_{0}-\lambda\right)$ is constant on a neighbourhood of $\lambda_{0}$, and hence it is 0 . Let $F$ be the finite rank operator defined by $F=I$ on $X_{1}, F=0$ on $X_{0}$. Hence, $A F=F A, \quad \alpha\left(A+F-\lambda_{0}\right)=0$ and $A+F-\lambda_{0} \in \Phi_{+}(X)\left(\left[7\right.\right.$, Theorem 5.26 of Chapter IV]). Thus, $\lambda_{0} \notin \sigma_{a}(A+F)$ and the proof is complete.

CoRollary 2.2. $\lambda \in \sigma_{a}(A) \backslash \sigma_{a b}(A)$ if and only if $\lambda$ is an isolated point of $\sigma_{a}(A)$, an eigenvalue of $A$ of finite multiplicity, $a(A-\lambda)<\infty$ and $R(A-\lambda)$ is closed.

Corollary 2.3. Let $\lambda \in \sigma_{a}(A)$ be an isolated point of $\sigma_{a}(A)$ and let $a(A-\lambda)=\infty$. Then $\lambda \in \sigma_{e a}(A)$.

Proof. Let $\lambda$ be an isolated point of $\sigma_{a}(A), a(A-\lambda)=\infty$ and $\lambda \notin \sigma_{e a}(A)$. Then $0<\alpha(A-\lambda)<\infty, R(A-\lambda)$ is closed and Kato's number $v(A-\lambda: I)$ is finite ([6, Theorem 3]). Let us apply the operator $F$ from the proof of Theorem 2.1. Then, by [3, Theorem 2], $a(A-\lambda)<\infty$, which provides a contradiction. This completes the proof.

COROLlary 2.4. $\sigma_{a b}(A)=\sigma_{e a}(A) \cup\left\{\right.$ limit points of $\left.\sigma_{a}(A)\right\}$.

Corollary 2.5. Let $A \in B(X)$. Then

(i) $\sigma_{e a}(A) \subset \sigma_{a b}(A) \subset \sigma_{e b}(A)$,

(ii) $\partial \sigma_{e b}(A) \subset \partial \sigma_{a b}(A) \subset \partial \sigma_{e a}(A)$,

(iii) $\hat{\sigma}_{e a}(A)=\hat{\sigma}_{a b}(A)=\hat{\sigma}_{e b}(A)$,

(iv) $\sigma_{a b}(A)\left(\sigma_{e b}(A)\right)$ can be obtained from $\sigma_{e a}(A)\left(\sigma_{a b}(A)\right)$ by filling in some holes of $\sigma_{e a}(A)\left(\sigma_{a b}(A)\right)$.

(v) If $\sigma_{e a}(A)$ is connected, $\sigma_{a b}(A)$ is connected, and if $\sigma_{a b}(A)$ is connected, $\sigma_{e b}(A)$ is connected.

Proof. It is sufficient to prove (ii). Since $\partial \sigma_{e b}(A) \subset \partial \sigma_{e m}(A)$ ([15, Theorem 1(b)]) and $\partial \sigma_{e m}(A) \subset \partial \sigma_{e a}(A)\left(\left[13\right.\right.$, Theorem 1]), then $\partial \sigma_{e b}(A) \subset \partial \sigma_{a b}(A)$. Suppose $\lambda_{0} \epsilon$ $\partial \sigma_{a b}(A)$ and $\lambda_{0} \notin \sigma_{e a}(A)$. Hence, $0<\alpha\left(A-\lambda_{0}\right)<\infty$ and $R\left(A-\lambda_{0}\right)$ is closed. Then there 
exists an $\varepsilon>0$ such that $0<\left|\lambda_{0}-\lambda\right|<\varepsilon$ implies that $R(A-\lambda)$ is closed and $\alpha(A-\lambda)$ is constant ([7, p. 243]). Since $\lambda_{0} \in \partial \sigma_{a b}(A)$, we have, by Theorem 2.1 , that the constant is 0 . Thus, $\lambda_{0}$ is an isolated point of $\sigma_{a}(A)$, and again by Theorem 2.1 we have that $a\left(A-\lambda_{0}\right)=\infty$. Hence, $\lambda_{0} \in \sigma_{e a}(A)$ (Corollary 2.3). This is a contradiction, and the proof is complete.

The following example was used by Salinas $([\mathbf{1 5}])$ in another context. We use it to show that in general $\sigma_{e a}(A) \neq \sigma_{a b}(A)$.

EXAMPLE 2.6. Let $H$ be a separable Hilbert space, and let $V$ be a unilateral shift of multiplicity one on $H$; also let $N \in B(H)$ be any quasinilpotent operator. Set $A=$ $V \oplus V^{*} \oplus N$. If we denote by $D$ the closed unit disc in $\mathbb{C}$ we have $\sigma_{a b}(A)=D$, while $\sigma_{e a}(A)=\partial D \cup\{0\}$.

Proof. Salinas showed that $\sigma_{e m}(A)=\partial D \cup\{0\}$ and $\sigma_{e b}(A)=D$. Hence, by $([13$, Theorem 2.1]) we have that $\sigma_{e a}(A)=\partial D \cup\{0\}$. Suppose $0<|\lambda|<1$ and $\lambda \notin \sigma_{a b}(A)$. Then $a(A-\lambda)<\infty$ (Theorem 2.1). Now $\lambda \notin \sigma_{e b}(A)$ ([16], [9, Theorem 1(4)]). This is a contradiction, and the proof is complete.

From the proof of Theorem 2.1 and Corollary 2.3 we have the following $\Phi_{+}^{-}(X)$-version of a T. J. Laffey and T. T. West theorem ([8, Proposition 2]).

Corollary 2.7. Let $A \in \Phi_{+}^{-}(X)$. Then the following statements are equivalent:

(i) $A=V+F$, where $\alpha(V)=0, F$ is finite rank and $V F=F V$;

(ii) there exists a finite rank projection $P$ commuting with $A$ such that $\alpha(A \mid N(P))=$ 0 ;

(iii) there exists $\varepsilon>0$ such that $\alpha(A+\lambda)=0$ for $0<|\lambda|<\varepsilon$;

(iv) $a(A)<\infty$.

\section{Spectral mapping theorem for $\sigma_{a b}(A)$.}

THEOREM 3.1. If $A$ is any operator and $p$ is any polynomial, then

$$
\sigma_{a b}(p(A))=p\left(\sigma_{a b}(A)\right) .
$$

Proof. Let $\lambda \notin p\left(\sigma_{a b}(A)\right)$ and $p(t)-\lambda=c\left(t-\lambda_{1}\right) \ldots\left(t-\lambda_{n}\right), c \neq 0$. Thus, $p(A)-\lambda$ $=c\left(A-\lambda_{1}\right) \ldots\left(A-\lambda_{n}\right)$, where $A-\lambda_{i} \in \Phi_{+}^{-}(X)$ and $a\left(A-\lambda_{i}\right)<\infty$ for $i=1, \ldots, n$ (Theorem 2.1). Then $p(A)-\lambda \in \Phi_{+}^{-}(X)$ ([17, Theorem 6.6, Theorem 3.5, Theorem 2.3 of Chapter V]). Let us show that $a(p(A)-\lambda)<\infty$. By $([5$, Proposition 38.7]) it is sufficient to prove that $p(A)-\lambda$ is injective on the subspace $U=\bigcap_{n=1}^{\infty}(p(A)-\lambda)^{n}(X)$. We shall use the method of mathematical induction. This is true for $n=1$. Suppose that this is true for all polynomials of degree $n-1$. Set $p(A)-\lambda=q(A)\left(A-\lambda_{1}\right)$ where $q(t)$ is polynomial of degree $n-1$. Let $x \in U$ and $(p(A)-\lambda) x=0$. Then $\left(A-\lambda_{1}\right)(q(A) x)=0$ and $q(A) x \in$ $\cap_{n=1}^{\infty}\left(A-\lambda_{1}\right)^{n}(X)$. Hence, by $([5$, Proposition 38.7]) $q(A) x=0$. Since $x \in$ $\bigcap_{n=1}^{\infty}(q(A))^{n}(X)$ and $q(t)$ is a polynomial of degree $n-1$, we have that $x=0$. Thus, we see by Theorem 2.1 that $\lambda \notin \sigma_{a b}(p(A))$. This shows that $\sigma_{a b}(p(A)) \subset p\left(\sigma_{a b}(A)\right)$. We now turn to the proof of the opposite inclusion. Suppose that $\lambda \in p\left(\sigma_{a b}(A)\right)$ and $\lambda \notin \sigma_{a b}(p(A))$. 
Then $p(A)-\lambda \in \Phi_{+}^{-}(X)$ and $a(p(A)-\lambda)<\infty$ (Theorem 2.1). By ([4, p. 20]) $A-\lambda_{i} \epsilon$ $\Phi_{+}(X)$ for $i=1, \ldots, n$. Let $\lambda_{j} \in \sigma_{a b}(A)$ and $\lambda=p\left(\lambda_{j}\right)$. Now, $\lambda$ is an isolated point of $\sigma_{a}(p(A))$ (Corollary 2.2) and by ([4]) $\lambda_{j}$ is an isolated point of $\sigma_{a}(A)$. Thus, $A-\lambda_{j} \in$ $\Phi_{+}^{-}(X)\left(\left[7\right.\right.$, Theorem 5.22 of Chapter IV]), and by Corollary 2.3 we have $a\left(A-\lambda_{j}\right)<\infty$. Again, by Theorem 2.1, we have that $\lambda_{j} \notin \sigma_{a b}(A)$, which provides a contradiction. This completes the proof.

To show that if $f$ is an analytic function defined on a neighbourhood of $\sigma(A)$, then $f\left(\sigma_{a b}(A)\right)=\sigma_{a b}(f(A))$ we shall apply K. Oberai's method ([12]). First we shall prove two following statements which are of particular interest.

THeOREM 3.2. Let $A \in B(X)$. Then the mapping $A \rightarrow \sigma_{a b}(A)$ is upper semicontinuous.

Proof. Let $A_{n} \rightarrow A$. We have to show that $\lim \sup \sigma_{a b}\left(A_{n}\right) \subset \sigma_{a b}(A)$. It is enough to show that if $0 \notin \sigma_{a b}(A)$, then $0 \notin \lim \sup \sigma_{a b}\left(A_{n}\right)$. Let $0 \notin \sigma_{a b}(A)$. Then 0 is an isolated point of $\sigma_{a}(A)$ and $A \in \Phi_{+}^{-}(X)$ (Theorem 2.1). By ([7, Theorem 5.22 of Chapter IV]) there exists an $\varepsilon>0$ and an integer $n_{1}$ such that $A_{n}-\lambda \in \Phi_{+}^{-}(X)$ for $|\lambda|<\varepsilon$ and for $n \geqslant n_{1}$. We may assume that $\alpha(A-\lambda)=0$ for $0<|\lambda|<\varepsilon$. Let $n \geqslant n_{1}$. Hence, by $([7$, p. 243]) $\alpha\left(A_{n}-\lambda\right)$ are constant for all $|\lambda|<\varepsilon$ except for an isolated set. Let $0<\left|\lambda_{0}\right|<\varepsilon$. Set $S_{0}=\left\{\lambda \in \mathbb{C}:|\lambda|=\left|\lambda_{0}\right|\right\}$ and $m(A)=\inf \{\|A x\|:\|x\|=1\}$. Now, $m(A-\lambda)>0$ for $\lambda \in S_{0}$. Since $m(A-\lambda)$ is a continuous function of $\lambda\left(\left[2\right.\right.$, p. 19]) and $S_{0}$ is compact, there exists a $\mu_{0} \in S_{0}$ such that $m\left(A-\mu_{0}\right)=\inf \left\{m(A-\lambda): \lambda \in S_{0}\right\}$. Let $n_{2}$ be an integer such that for $n \geqslant n_{2}$ we have $\left\|A_{n}-A\right\|<m\left(A-\mu_{0}\right)$. Hence, for $n \geqslant n_{0}=\max \left(n_{1}, n_{2}\right)$ and $\lambda \in S_{0}$ we have $m(A-\lambda)-\left\|A-A_{n}\right\| \leqslant m\left(A_{n}-\lambda\right) \quad\left(\left[2\right.\right.$, Lemma 2.2]). Thus, $m\left(A_{n}-\lambda\right)>0$ and $\alpha\left(A_{n}-\lambda\right)=0$ for all $|\lambda|<\varepsilon$ except for an isolated set and for $n \geqslant n_{0}$. Therefore, for $n \geqslant n_{0}$ we see that $\sigma_{a b}\left(A_{n}\right) \cap\{\lambda \in \mathbb{C}:|\lambda|<\varepsilon\}$ is empty (Corollary 2.3 and Theorem 2.1). Thus we have $0 \notin \lim \sup \sigma_{a b}\left(A_{n}\right)$, and the proof is complete.

THeOREM 3.3. Let $A \in B(X)$ and let $f$ be an analytic function defined on a neighbourhood of $\sigma(A)$. Then

$$
\sigma_{e a}(f(A)) \subset f\left(\sigma_{e a}(A)\right) \text {. }
$$

Proof. $\Phi_{+}(X)$ is an $F$-semigroup with index $i([4$, p. 20]). Also

$$
\sigma_{e a}(A)=\sigma_{e \alpha}(A) \cup\left(\bigcup_{n \geq 1} F_{n}\right),
$$

where $\sigma_{e \alpha}(A)=\left\{\lambda \in \mathbb{C}: A-\lambda \notin \Phi_{+}(X)\right\}, \quad F_{n}=\left\{\lambda \in \sigma(A): A-\lambda \in \Phi_{+}(X), \quad i(A-\lambda)=n\right\}$. Now suppose that $\mu \notin f\left(\sigma_{e a}(A)\right)$. Then $\mu-f(\lambda)$ has no zeros on $\sigma_{e a}(A)$ and in particular has no zeros on $\sigma_{e \alpha}(A)$. Applying $([4$, Theorem 1]) we conclude that $\mu-f(A) \in \Phi_{+}(X)$ and

$$
i(\mu-f(A))=\sum_{n} n \alpha_{n},
$$

where $\alpha_{n}$ is the number of isolated zeros of $\mu-f(\lambda)$ on $F_{n}$ counted according to their multiplicities. From (3.1) it follows that $\alpha_{n}=0$ for $n \geqslant 1$. Thus $i(\mu-f(A)) \leqslant 0$, which implies that $\mu \notin \sigma_{e a}(f(A))$. This completes the proof. 
THEOREM 3.4. Let $A \in B(X)$ and let $f$ be an analytic function defined on a neighbourhood of $\sigma(A)$. Then

$$
f\left(\sigma_{a b}(A)\right)=\sigma_{a b}(f(A)) \text {. }
$$

Proof. Let $\left(p_{n}(t)\right)$ be a sequence of polynomials converging uniformly to $f(t)$ on a neighbourhood of $\sigma(A)$. We have

$$
\begin{aligned}
f\left(\sigma_{a b}(A)\right) & =\lim p_{n}\left(\sigma_{a b}(A)\right) & & (\text { by }([\mathbf{1 2}, \mathrm{p} .370]) \\
& =\lim \sigma_{a b}\left(p_{n}(A)\right) & & (\text { by Theorem 3.1) } \\
& \subset \sigma_{a b}(f(A)) & & \text { (by Theorem 3.2). }
\end{aligned}
$$

To prove the converse suppose that $\lambda \in \sigma_{a b}(f(A))$.

Case I. $\lambda \in \sigma_{e a}(f(A))$. By Theorem $3.3 \lambda \in f\left(\sigma_{e a}(A)\right)$. Thus, we have $\lambda \in f\left(\sigma_{a b}(A)\right)$.

Case II. $\lambda \notin \sigma_{e a}(f(A))$. In this case $\lambda$ is a limit point of $\sigma_{a}(f(A))$ (Corollary 2.4), and there exists a sequence $\left(\lambda_{n}\right) \subset \sigma_{a}(f(A))$ such that $\lambda_{n} \rightarrow \lambda$. Now, there exists a sequence $\left(\mu_{n}\right) \in \sigma_{a}(A)$ such that $f\left(\mu_{n}\right)=\lambda_{n} \rightarrow \lambda([4])$. Then $\left(\mu_{n}\right)$ contains a convergent subsequence and we may assume that $\lim \mu_{n}=\mu \in \sigma_{a}(A)$. Then $\lambda=f(\mu) \in f\left(\sigma_{a b}(A)\right)$. This completes the proof of the theorem.

\section{A perturbation theorem.}

Theorem 4.1. Let $A \in B(X)$ and let $N \in B(X)$ be a quasinilpotent operator commuting with $A$. Then $\sigma_{a b}(A+N)=\sigma_{a b}(A)$.

Proof. It is enough to show that if $0 \notin \sigma_{a b}(A)$, then $0 \notin \sigma_{a b}(A+N)$. Let $0 \notin \sigma_{a b}(A)$. If $0 \notin \sigma_{a}(A)$, then $0 \notin \sigma_{a}(A+N)\left(\left[1\right.\right.$, p. 320]). Hence, we have that $0 \notin \sigma_{a b}(A+N)$. If $0 \in \sigma_{a}(A)$, then 0 is an isolated point of $\sigma_{a}(A)$ (Corollary 2.2), and therefore 0 is an isolated point $\sigma_{a}(A+N)([1$, p. 320$])$. Since $0 \notin \sigma_{a b}(A)$, we see that $0 \notin \sigma_{e a}(A+N)([19$, Theorem 7]). This implies that $0 \notin \sigma_{a b}(A+N)$ (Corollary 2.3), and the proof is complete.

ACKNowledgement. I am grateful to Dr. J. Zemánek for his interest in this paper and for a useful remark in connection with the proof of Theorem 2.1.

\section{REFERENCES}

1. M. D. Choi and C. Davis, The spectral mapping theorem for joint approximate point spectrum, Bull. Amer. Math. Soc. 80 (1974), 317-321.

2. H. A. Gindler and A. E. Taylor, The minimum modulus of a linear operator and its use in spectral theory, Studia Math. 22 (1962), 15-41.

3. S. Grabiner, Ascent, descent, and compact perturbations, Proc. Amer. Math. Soc., 71 (1978), 79-80.

4. B. Gramsh and D. Lay, Spectral mapping theorems for essential spectra, Math. Ann., 192 (1971), 17-32.

5. H. G. Heuser, Functional analysis (Wiley-Interscience, 1982).

6. T. Kato, Perturbation theory for nullity, deficiency and other quantities of linear operators, J. Analyse Math., 6 (1958), 261-322.

7. T. Kato, Perturbation theory for linear operators (Springer-Verlag, 1976). 
8. T. J. Laffey and T. T. West, Fredholm commutators, Proc. Roy. Irish Acad. Sect. A, 82 (1982), 129-140.

9. D. Lay, Characterizations of the essential spectrum of F. E. Browder, Bull. Amer. Math. Soc., 74 (1968), 246-248.

10. D. Lay, Spectral analysis using ascent, descent, nullity and defect, Math. Ann., 184 (1970), 197-214.

11. K. K. Oberai, On the Weyl spectrum, Illinois J. Math., 18 (1974), 208-212.

12. K. K. Oberai, Spectral mapping theorem for essential spectra, Rev. Roumaine Math. Pures Appl., 25 (1980), 365-373.

13. V. Rakočević, On one subset of M. Schechter's essential spectrum, Mat. Vesnik, 5 (1981), 389-391.

14. V. Rakočević, On the essential approximate point spectrum II, Mat. Vesnik, 36 (1984), 89-97.

15. N. Salinas, Operators with essentially disconnected spectrum, Acta Sci. Math. (Szeged), 33 (1972), 193-205.

16. M. Schechter, On the essential spectrum of an arbitrary operator I, J. Math. Anal. Appl., 13 (1966), 205-215.

17. M. Schechter, Principles of functional analysis (Academic Press, Student edition, 1973).

18. J. Zemánek, Geometric characteristic of semi-Fredholm operators and their asymptotic behaviour, Institute of Mathematics, Polish Academy of Sciences, preprint 290 (1983).

19. J. Buoni, R. Harte and T. Wickstead, Upper and lower Fredholm spectra, Proc. Amer. Math. Soc., 66 (1977), 309-314.

UNIVERSITY OF NISH

FaCUlTy of Philosophy

Department of Mathematics

Cirila and Metodija 2

18000 NISH

YUGOSLAVIA 